\title{
Metabolic changes during carcinogenesis: Potential impact on invasiveness
}

\author{
Kieran Smallbone, ${ }^{\mathrm{a}, *}$, Robert A. Gatenby ${ }^{\mathrm{b}}$, Robert J. Gillies ${ }^{\mathrm{b}}$, \\ Philip K. Maini ${ }^{\mathrm{a}}$, David J. Gavaghan ${ }^{\mathrm{c}}$ \\ ${ }^{a}$ Centre for Mathematical Biology, Mathematical Institute, 24-29 St Giles', Oxford, OX1 3LB, UK \\ ${ }^{\mathrm{b}}$ Department of Radiology, University of Arizona, 1501 N. Campbell Avenue, Tucson, AZ 85724-5067, USA \\ ${ }^{\mathrm{c}}$ Oxford University Computing Laboratory, Wolfson Building, Parks Road, Oxford, OX1 3QD, UK
}

Received 20 June 2006; received in revised form 29 August 2006; accepted 7 September 2006

Available online 16 September 2006

\begin{abstract}
Successful adaptation to varying microenvironmental constraints plays a crucial role during carcinogenesis. We develop a hybrid cellular automation approach to investigate the cell-microenvironmental interactions that mediate somatic evolution of cancer cells. This allows investigation of the hypothesis that regions of premalignant lesions develop a substrate-limited environment as proliferation carries cells away from blood vessels which remain separated by the intact basement membrane. We find that selective forces in tumoural regions furthest from the blood supply act to favour cells whose metabolism is best suited to respond to local changes in oxygen, glucose and $\mathrm{pH}$ levels. The model predicts three phases of somatic evolution. Initially, cell survival and proliferation is limited due to diminished oxygen levels. This promotes adaptation to a second phase of growth dominated by cells with constitutively up-regulated glycolysis, less reliant on oxygen for ATP production. Increased glycolysis induces acidification of the local environment, limiting proliferation and inducing cell death through necrosis and apoptosis. This promotes a third phase of cellular evolution, with emergence of phenotypes resistant to acid-induced toxicity. This emergent cellular phenotype has a significant proliferative advantage because it will consistently acidify the local environment in a way that is toxic to its competitors but harmless to itself. The model's results suggest this sequence is essential in the transition from self-limited premalignant growth to invasive cancer, and, therefore, that this transition may be delayed or prevented through novel strategies directed towards interrupting the hypoxia-glycolysis-acidosis cycle.
\end{abstract}

(C) 2006 Elsevier Ltd. All rights reserved.

Keywords: Carcinogenesis; Glycolytic phenotype; Mathematical modelling

\section{Introduction}

Increased glycolysis is characteristically observed in invasive cancers. In part this is due to development of intratumoural regions of hypoxia, arising from disordered vascular development and flow. However, it is also the result of persistent anaerobic metabolism even in the presence of oxygen (aerobic glycolysis) (Warburg, 1930). The inefficiency of anaerobic metabolism is compensated

\footnotetext{
${ }^{*}$ Corresponding author. Present address: Manchester Centre for Integrative Systems Biology, Manchester Interdisciplinary Biocentre, 131 Princess Street, Manchester, M1 7DN, UK; Tel.: + 441613065197 ; fax: + 441613068918 .

E-mail address: kieran.smallbone@manchester.ac.uk (K. Smallbone).
}

by a several-fold increase in glucose flux. This phenomenon is now routinely exploited for tumour imaging through FDG-PET $\left({ }^{18}\right.$ fluorodeoxyglucose positron emission tomography) (Czernin and Phelps, 2002; Gambhir, 2002). PET has confirmed that the vast majority $(>90 \%)$ of human primary and metastatic tumours demonstrate increased glucose uptake indicating abnormal metabolism. Furthermore, PET has been used to show a direct correlation between tumour aggressiveness and the rate of glucose consumption (Di Chiro et al., 1987).

The almost universal presence of aerobic glycolysis in the phenotype of such a wide range of cancers arising in multiple different sites seems inconsistent with the evolutionary model of carcinogenesis. That is, invasive cancers appear to arise through a complex multi-step process often 
described as somatic evolution because it is governed by continuous heritable changes in the tumour populations and environmental selection pressures that promote proliferation of phenotypes best adapted to their microenvironment. Due to these Darwinian dynamics, it is reasonable to assume the common appearance of a specific phenotype within a large number of different cancer populations is evidence that it must confer a significant growth advantage. However, the proliferative advantages gained from altered glucose metabolism are far from clear. Firstly, anaerobic respiration is more than an order of magnitude less efficient than its aerobic counterpart, producing only 2 ATP per glucose in comparison to approximately 36 ATP. Secondly, the $\mathrm{H}^{+}$(hydrogen ions) produced as a result of glycolysis cause a consistent acidification of the extracellular space that is potentially toxic (Schornack and Gillies, 2003). In particular, an acidic microenvironment results in tissue damage due to cell death and degradation of the extracellular matrix (Rohzin et al., 1994). Intuitively, one would expect the Darwinian forces prevailing during carcinogenesis to select against this inefficient and environmentally toxic phenotype, in favour of more optimal metabolic regimes. Thus, the evolutionary dynamics that leads to adoption of aerobic glycolysis as a typical component of the malignant phenotype remains unknown.

Gatenby and Gillies (2004) propose that evolution of aerobic glycolysis is the result of environmental constraints imposed by the morphology of the ducts in which premalignant lesions evolve. Specifically, they point out that the epithelium in ducts remains separated from the blood supply by the intact basement membrane. Initial proliferation in premalignant lesions carries cells into the lumen, away from the basement membrane, and, therefore, away from their blood supply. This steadily increases the distance that substrate must diffuse between the vessels and the intraluminal tumour cells and results in regions of hypoxia but near normal glucose concentrations. They proposed this initiates an evolutionary sequence consisting of adaptation to hypoxia by upregulation of glycolysis, acidification of the environment due to anaerobic respiration of glucose, and then cellular adaptation to acidinduced cellular toxicity. They pointed out that the phenotype that emerges from this sequence has a powerful adaptive advantage because it creates an environment (due to increased glycolysis) that is toxic to its competitors but relatively harmless to itself. This adaptive advantage may be sufficient to allow unconstrained proliferation and, thus, be a critical component in the transition from a premalignant tumour to an invasive cancer.

This model is supported by experimental observations of upregulation of cellular responses to hypoxia in regions of premalignant DCIS (ductal carcinoma in situ) and PIN (intraepithelial neoplasia) most distant from the basement membrane. This includes upregulation of HIF (hypoxiainducible factor) and related proteins such as carbonic anhydrase IX and GLUT-1 (glucose transporter 1) (Kunkel et al., 2003; Wykoff et al., 2001). However, it is clear that in vivo experimental verification of the hypothesis that the final stages of carcinogenesis are driven by cellular adaptation to hypoxia and acidosis will be difficult.

Experience in the physical sciences has demonstrated that the dynamics that govern complex, multi-scale systems such as carcinogenesis cannot be fully captured by linear, intuitive word models and, instead, require detailed mathematical modelling (Gatenby et al., 2002; Gatenby and Maini, 2003; Komarova, 2005). To test the feasibility of the theoretical model of Gatenby and Gillies (2004), we frame the hypothesis using mathematical methods that examine somatic evolution of premalignant cells within the constraints of ductal anatomy. This allows us to test the proposed sequence of environmental changes and cellular adaptations in silico. We accomplish this using evolutionary models of carcinogenesis that explicitly include spatial parameters to accommodate the geometry of early tumour development. This requires application of a hybrid cellular automaton approach (Anderson, 2005; Patel et al., 2001). The key advantage of this technique is that it allows cells to be treated as discrete individuals, enabling cellular processes such as proliferation, death, adaptation and metabolite consumption/production to be modelled at the individual cell level. However, the automaton is described as hybrid because the metabolite distributions, specifically the oxygen, glucose and $\mathrm{H}^{+}$concentrations, are allowed to form a continuous field across the cells.

Analysis of the model gives us useful insight into the role of the microenvironment in mediating the somatic evolution of cancer cells. In turn, novel therapeutic strategies are suggested for inhibiting the emergence of the glycolytic phenotype and hence slowing the progression of early lesions.

\section{Materials and methods}

A hybrid cellular automaton model is used to simulate carcinogenesis. This two-dimensional model is composed of an $M \times N$ array of automaton elements $(i, j)$ with a specific rule-set governing their evolution, as well as oxygen, glucose and $\mathrm{H}^{+}$fields, each satisfying reactiondiffusion equations. Each automaton element corresponds to either a tumour cell or a vacant space. Tumour cell diameter can be highly variable, ranging from 10 to $100 \mu \mathrm{m}$ (Melicow, 1982), depending on the specific tumour type under consideration. Here we assume each automaton element, and hence each tumour cell, has constant physical size $\Delta \times \Delta$, where $\Delta=25 \mu \mathrm{m}$.

The phenotypic traits of malignant cancers arise as a result of environmental selection pressures during carcinogenesis (Bernards and Weinberg, 2002). Hence it is important to understand the physical environment of early pre-malignant lesions. Carcinomas in situ are often characterized as highly vascularized. This is misleading, however, as whilst they may have a vascular stroma (external connective tissue), the tumour cells are actually physically separated from their blood supply by a thin 

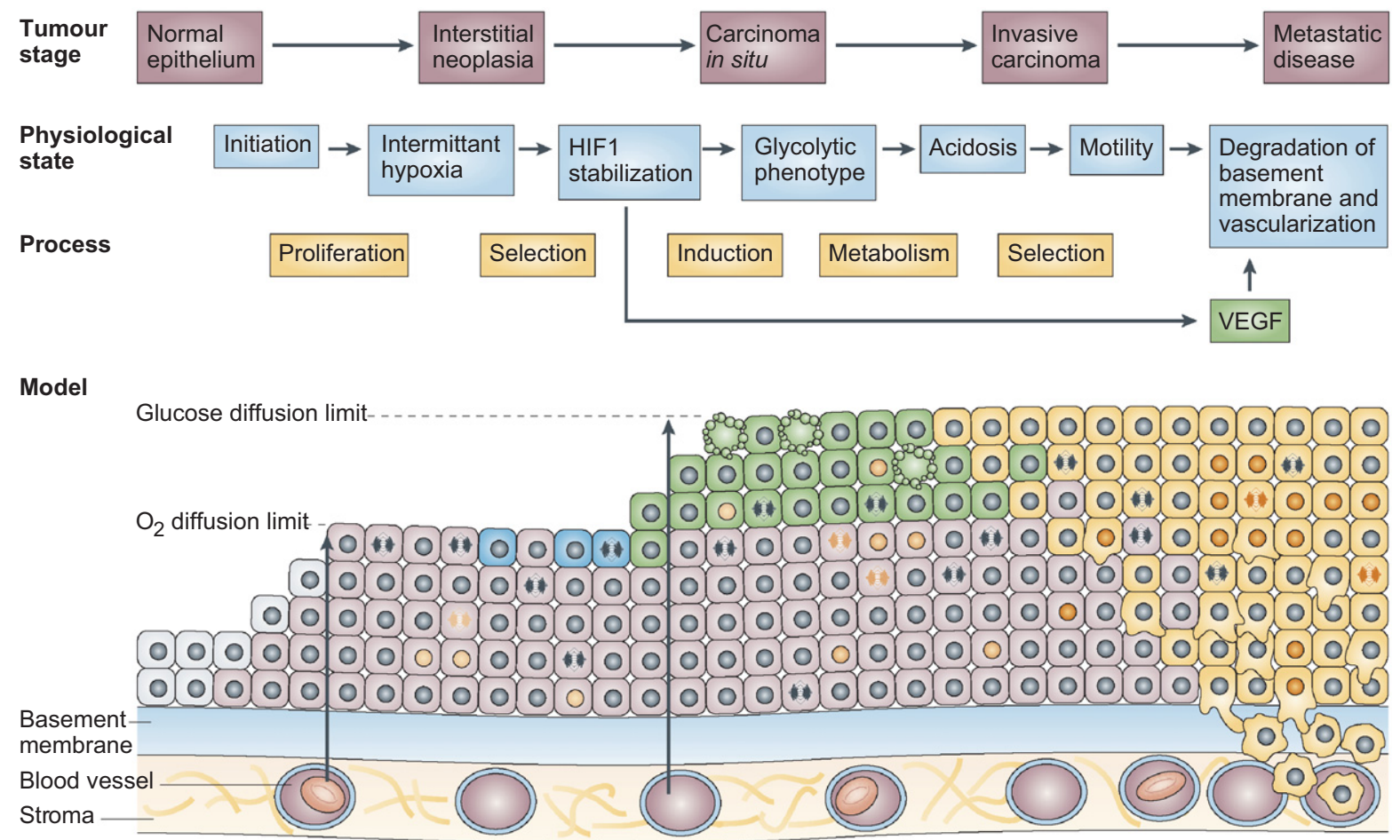

Fig. 1. Model for cell-environment interactions during carcinogenesis, giving the stages of tumour growth and their associated physiological states. Shown are normal epithelial (grey), hyperplastic (pink), hypoxic (blue), glycolytic (green) and motile (yellow) cells. Reproduced with permission from Nature Reviews Cancer (Gatenby and Gillies, 2004) (C2004 Macmillan Magazines Ltd.

basement membrane, until this membrane is breached by an invasive cell. Therefore, carcinogenesis and the development of the malignant phenotype actually occur in an avascular environment, whereby substrates must diffuse across the basement membrane and through layers of tumour cells to be metabolized. This anatomy places consistent and significant boundary conditions on the biology of carcinogenesis. In the model we reflect this geometry by assuming that the bottom edge of the array $i=0$ represents the basement membrane. Beyond the membrane we assume the stroma is sufficiently wellvascularized that the metabolites remain at their normal extracellular concentrations.

A model for the key cell-environment interactions we propose to occur during carcinogenesis is shown in Fig. 1. Initially, normal epithelial cells grow along the basement membrane, with the epithelial layer at most a few cells thick. Homeostasis mechanisms do not normally allow growth of these cells away from the basement membrane. However, following initial genetic events in the carcinogenesis pathways such as those depicted by the Fearon-Vogelstein model (Fearon and Vogelstein, 1990), the cells become hyperplastic, leading to a thickening of the epithelial layer, pushing cells into the lumen and away from the basement membrane. Since the blood vessels remain outside the basement membrane, nutrients and waste must diffuse over longer and longer distances. As a result, it is likely that hyperplastic cells beyond the Thomlinson-Gray limit of 100-150 $\mathrm{m}$ (Thomlinson and Gray, 1955) from the basement membrane will experience profound hypoxia, which will initiate a sequence of critical cellular adaptations and environmental changes. Specifically, it is proposed that hypoxia leads to constitutive upregulation of glycolysis which, in turn, results in increased $\mathrm{H}^{+}$ production and acidification of the microenvironment. This decreased extracellular $\mathrm{pH}\left(\mathrm{pH}_{e}\right)$ is toxic to the local populations because it induces $\mathrm{p} 53$-dependent apoptosis due to increased caspase activity. This selects for cells that are resistant to acid-induced toxicity resulting in further evolution of new phenotypic properties that, for example, increase the number and activity of $\mathrm{Na}^{+} / \mathrm{H}^{+}$antiporters on the cell surface or possess mutations in p53, caspase or other components of the acid-induced apoptosis pathways. The acidosis also selects for motile cells that eventually breach the basement membrane, gaining access to existing and newly formed blood and lymphatic routes for metastasis.

To investigate this hypothesis, we consider the selective pressures placed on a number of different possible tumour phenotypes. Initially, the automaton consists of a layer of a normal epithelial tissue. We assume it to be a simple epithelium i.e. the cells grow in a monolayer along the basement membrane. Then the initial array consists of normal cells at $(1, j)$ and is vacant elsewhere. As well as proliferation and death, we assume that these cells may randomly undergo three possible heritable changes, either through mutations or epigenetic changes such as alterations in the methylation patterns of promoters. The cells may become:

- hyperplastic, allowing growth away from the basement membrane; 
- glycolytic, increasing their rate of glucose uptake and utilization;

- acid-resistant, requiring a lower extracellular $\mathrm{pH}$ to induce toxicity.

These three changes give rise to eight different phenotype combinations, and thus eight competing cellular populations. The time-scales for induction of the three heritable changes during carcinogenesis will give insight as to their relative importance.

\subsection{Cellular metabolism}

We first define a simple model of cellular glucose metabolism. Under normal physiological conditions, human cells rely on aerobic respiration to produce their energy. Each glucose molecule reacts with six oxygen molecules to produce carbon dioxide and ATP. This reaction may be caricatured by

glucose $+6 \mathrm{O}_{2} \longrightarrow 6 \mathrm{CO}_{2}+n_{A} \mathrm{ATP}$,

where $n_{A}$ denotes the number of ATP molecules produced during complete oxidation of glucose. In this paper we assume $n_{A}=36$, though this value may vary slightly depending on the specific cell type under consideration.

During periods of hypoxia, cells revert to the less efficient anaerobic metabolism, producing two molecules of lactic acid per glucose,

glucose $\longrightarrow$ 2lactic acid +2 ATP.

The details of the mathematical models capturing cellular dynamics are included in the Appendix. During the first stage of carcinogenesis, the dominant growth constraints involve cellular interactions with the extracellular matrix and other cells. During this phase, substrate supplies are assumed adequate and, therefore, cellular metabolism neither promotes nor constrains growth. Once these social constraints have been overcome and tumour cells proliferate into the lumen and away from the basement membrane, the dominant growth constraint becomes limited substrate availability, and thus increased ATP production confers a competitive advantage.

The dynamics of aerobic and anaerobic metabolism of glucose are summarized in Eqs. (9)-(12) in the Appendix. Cells using inefficient glycolytic metabolism maintain adequate ATP concentrations by increasing glucose flux. Normal cells are assumed to adopt glycolytic metabolism only when environmental conditions are hypoxic (the Pasteur effect, Racker, 1974). Transformed cells exhibit a similar response to hypoxia but also maintain glycolytic metabolism even in the presence of oxygen (the Warburg effect, Warburg, 1930). Differences between the two cell types are also seen in $\mathrm{H}^{+}$production. Normal cells produce increased acid (i.e. above the basal rate) only when oxygen supply is low. However, glycolytic cells produce increased amounts of $\mathrm{H}^{+}$even in normoxic conditions and thus acidify the extracellular space, irrespective of the oxygen levels.

\subsection{Metabolite profiles}

Having defined a model of cellular respiration, we are now in a position to determine the metabolite distributions around the cells. The details of the mathematical modelling are included in the Appendix. After each automaton generation, the known rates of metabolite consumption and production for each cell are used to calculate the corresponding metabolite profiles. This allows us to generate a continuously varying regional map of oxygen, glucose, and acid concentrations.

\subsection{Cell dynamics}

We now proceed to investigate how the carcinoma evolves in response to the associated distribution of glucose, oxygen and $\mathrm{H}^{+}$within the tissue. Initially, the automaton is composed of normal cells forming a monolayer along the basement membrane. After each generation, the resultant glucose, oxygen and $\mathrm{H}^{+}$fields are calculated using the methods outlined above. Each cell in the automaton is then updated (in a random order) according to the local metabolite levels. Cells may proliferate, adapt or die, and cells with different phenotypic patterns respond to the microenvironmental pressures in different ways. As such, competition is incorporated into the model: for a new population to progress and grow, it must successfully compete for space and resources with existing populations.

The rules governing the evolution of the automaton elements are as follows:

(1) An element that is empty does not evolve directly. It may evolve indirectly when cell division takes place in a neighbouring cell.

(2) If the amount of ATP produced by a cell $\phi_{a}$ falls below a critical threshold value, $a_{0}$, it dies, and the element becomes empty. As such, $a_{0}$ represents the level of ATP required for normal cellular maintenance. We do not allow hypoxia to directly induce cellular death within our model. Rather, hypoxia indirectly causes cell death through a reduction in ATP production. As mentioned previously, cells displaying the glycolytic phenotype produce significantly more ATP than their normal counterparts during periods of hypoxia, thus they are less susceptible to cell death via this mechanism. We assume $a_{0}=0.1$, corresponding to normal cell death occurring when oxygen levels drop below $c=0.05$ (Anderson, 2005).

(3) The local $\mathrm{H}^{+}$level, $h$ may also induce cellular death, with probability $p_{\text {dea }}$. We define this probability by

$$
p_{\text {dea }}= \begin{cases}h / h_{N} & \text { in a normal cell, if } h<h_{N}, \\ h / h_{T} & \text { in an acid-resistant cell, if } h<h_{T}, \\ 1 & \text { otherwise, }\end{cases}
$$


where $h_{N}<h_{T}$. Thus the probability of cell death increases with acidity, and the cell will always die if the $\mathrm{H}^{+}$level is greater than $h_{N}$ or $h_{T}$, dependent on the cell type under consideration. These values are taken to be $h_{N}=9.3 \times 10^{2}$ and $h_{T}=8.6 \times 10^{3}$ for normal and acid-resistant cells, respectively, corresponding to threshold values of $\mathrm{pH} 6.8$ and 6 (Patel et al., 2001).

(4) If the cell is not attached to the basement membrane, and is not hyperplastic, it dies.

(5) If the cell does not die through any of the mechanisms above, it either attempts to divide, with probability $p_{\text {div }}$, or becomes quiescent. The probability of division is a function of the cellular ATP production

$p_{\text {div }}= \begin{cases}\left(\phi_{a}-a_{0}\right) /\left(1-a_{0}\right), & a_{0}<\phi_{a}<1, \\ 1, & \phi_{a} \geqslant 1 .\end{cases}$

Hence, we assume that the probability of division is proportional to the ATP generated that is not needed for maintenance, and that the cell will always attempt to divide if the production rate is more than its normal level of one. If the cell attempts to divide, we determine whether cell division occurs by sampling its neighbouring elements. If there is one empty space, then the cell divides, and the new cell occupies this empty space. If there is more than one empty space, the new cell goes to the element with the largest oxygen concentration (following Alarcón et al., 2003).

(6) If a cell divides, each of the two daughter cells has probability $p_{a}$ of randomly acquiring one of the three heritable characteristics (hyperplasia, glycolysis and acid resistance). In order to avoid bias in the model, we assume these changes are reversible. For example, a cell displaying constitutive up-regulation of glycolysis may revert to normal glucose metabolism; if this metabolism is most appropriate for the current microenvironmental conditions, the cell will successfully compete for resources with its neighbours. We choose $p_{a}=10^{-3}$ as a base value, to reflect the fact that heritable change is a relatively rare occurrence.

It remains to define the dimensions of the automaton $M$ and $N$. We take $N=50$, corresponding to a typical ductal carcinoma of radius $200 \mu \mathrm{m}$. However, we leave $M$ undefined, allowing it to dynamically increase as the carcinoma grows. Essentially the final value taken by $M$ will represent the maximum distance from the basement membrane the cells may survive, given the limited nutrient supply and acid removal.

Throughout this model derivation, we have assumed that various processes follow simple, linear dynamics (Eqs. (3), (4), (7) and (8)). It can be argued that these assumptions are too unrealistic to represent complex biological phenomena such as these. However, these processes are poorly understood and, as a first approximation, an assumption of linearity is sufficient to capture qualitatively similar monotonic behavior. We would not expect these assumptions to have a marked effect on the model's conclusions.
Moreover, the relative simplicity of the model means that the parameter space is kept to a manageable size.

\section{Results}

We now apply the procedures outlined in the previous section. The simulations involved systematically varying the glycolytic rate $k$, tumour cell acidity threshold $h_{T}$ and adaptation rate $p_{a}$ whilst keeping other parameters constant. Multiple repetitions of the evolution of the system for each $\left(k, h_{T}, p_{a}\right)$ triple were performed to obtain adequate statistics.

Fig. 2 shows the temporal evolution of a typical cellular automaton $\left(k=10, h_{T}=8.6 \times 10^{3}, p_{a}=10^{-3}\right)$, and may be compared to the model described in Fig. 1. Initially, normal epithelial cells (grey) line the basement membrane (Fig. 2(a)). Acquisition of the hyperplastic phenotype (pink) allows growth away from the membrane towards the oxygen diffusion limit (Fig. 2(b)). Beyond this point, cells cannot exist as the oxygen levels are insufficient to meet cellular ATP demands. This drives adaptation to a glycolytic phenotype (green), less reliant on oxygen for ATP production (Fig. 2(c)). The increased ATP levels within glycolytic cells give a competitive advantage over the existing population, thus glycolytic cells dominate the system. Note, however, that the total number of cells within the system has decreased; the increased reliance on glycolysis has resulted in higher levels of acidity, in turn inducing cell death. Further adaptation occurs to an acidresistant phenotype (Fig. 2(d)). Increased use of glycolysis allows growth well beyond the oxygen diffusion limit, whilst the cells are more resistant to the resulting acidosis.

Fig. 3 shows how metabolite levels vary across the lesion at this final stage of development. Here we see growth approximately 30 cells deep from the basement membrane. Oxygen levels drop to $c=0.02$, in comparison to their normal level of one. In contrast, glucose levels fall to $g=0.9$; despite the ten-fold increase in consumption rate, the extracellular glucose levels are only slightly reduced. This is an important point - over the length scale of carcinogenesis, glucose supply is not a limiting factor. Rather, the cells furthest from the basement membrane are kept at equilibrium through a modest reduction in ATP production $\left(\phi_{a}=0.5\right)$ accompanying cellular death through a large increase in $\mathrm{H}^{+}$levels $\left(h=2 \times 10^{3}\right)$. In contrast to the theoretical model presented in Fig. 1, we find that the most likely mechanism for necrosis of cells furthest from the basement membrane is acid-induced toxicity, rather than glucose deprivation. In turn, this enhances the argument that acid-induced cellular toxicity is a major evolutionary force in the hypoxic regions of premalignant tumours. The inhibitory effect conferred by acidosis increases with distance from the basement membrane, inducing heterogeneities that may be seen in Fig. 2(b)-(d).

In Fig. 4 the proportion of cells displaying each heritable change is shown for the automaton displayed in Fig. 2. 

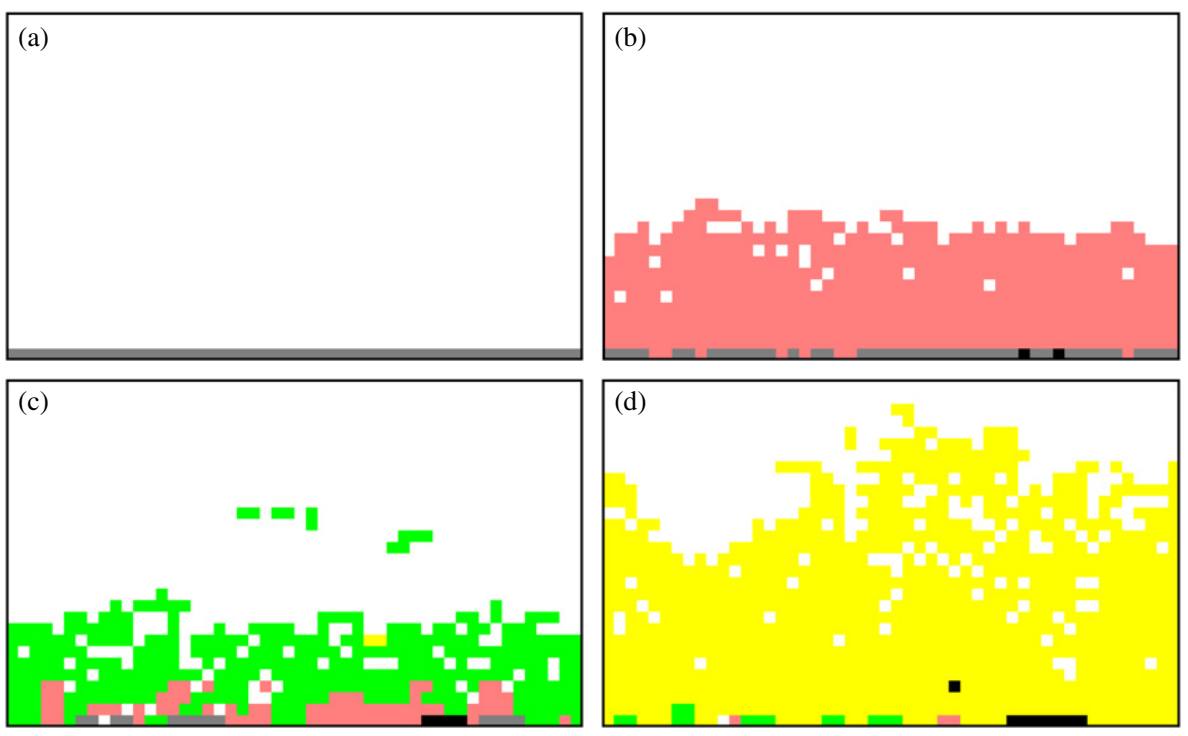

Fig. 2. The temporal evolution of a typical cellular automaton $\left(k=10, h_{T}=8.6 \times 10^{3}, p_{a}=10^{-3}\right.$ ) after (a) $t=0$, (b) $t=100$, (c) $t=250$ and (d) $t=300$ generations. Shown are normal epithelial (grey), hyperplastic (pink), hyperplastic-glycolytic (green) and hyperplastic-glycolytic-acid-resistant (yellow) cells. Cells with other phenotypic patterns are shown as black.

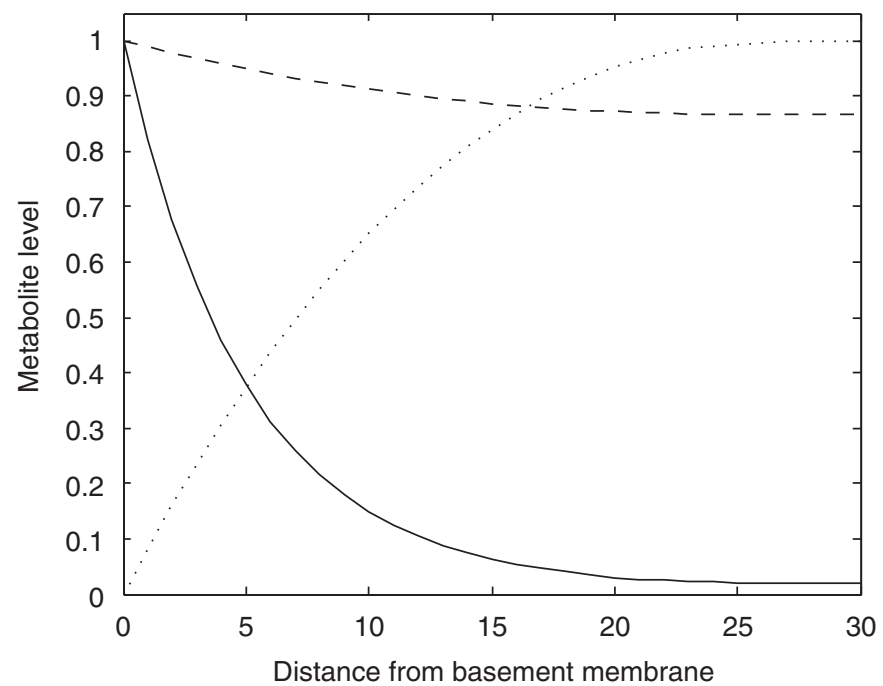

Fig. 3. Variation in metabolite concentrations with distance from the basement membrane, for the automaton generation displayed in Fig. 2(d). Shown are the mean glucose (dashed line), oxygen (solid line) and $\mathrm{H}^{+}$ (dotted line) concentrations. The $\mathrm{H}^{+}$profile has been scaled by its maximum value $\left(h \approx 2 \times 10^{3}\right)$.

The three stages of growth from normal cells acquiring, in turn, hyperplastic, glycolytic and acid-resistant phenotypes can be clearly seen. The steepness of the hyperplastic and acid-resistant curves suggests that these changes are extremely beneficial to the underlying population. The glycolytic curve is shallower as the benefits of increased ATP production are counteracted by acidosis. The order in which changes are accumulated is random; however, for a new phenotype to successfully compete with an existing population for resources it must be better suited to respond to existing micro-environmental factors. It is interesting to note that throughout the simulations performed here, the

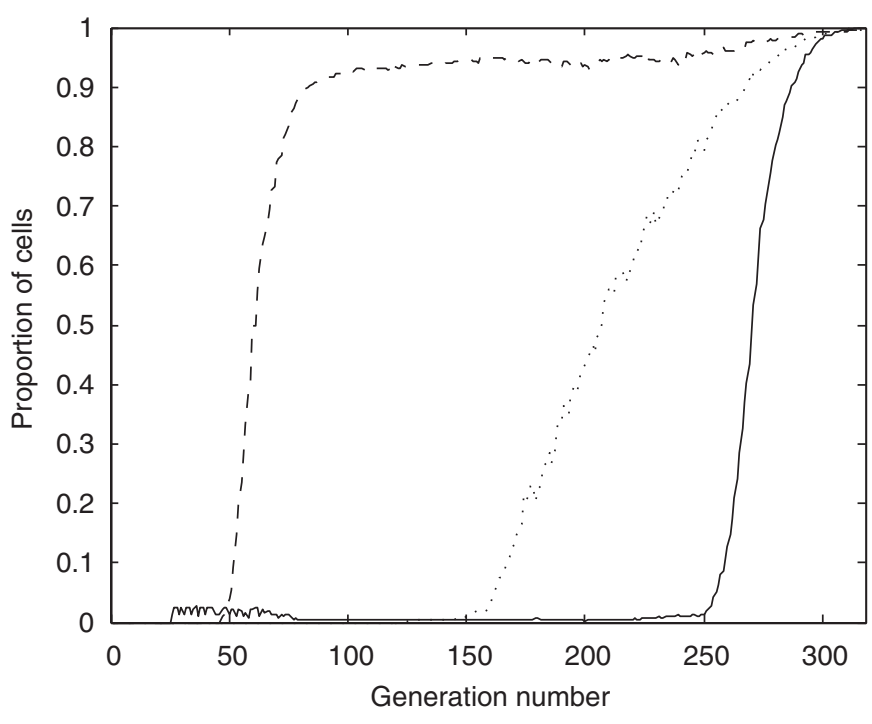

Fig. 4. Accumulation of heritable changes over time, for the automaton displayed in Fig. 2. Shown are the proportion of cells displaying the hyperplastic (dashed line), glycolytic (dotted line) and acid-resistant (solid line) phenotypes.

heritable changes within the dominant population are accumulated in this same order. Within our model, the underlying environmental selection parameters drive the cells to always follow this adaptive pathway - escaping in turn from the constraints of limited proliferation (hyperplasia), substrate availability (glycolysis) and waste removal (acid resistance). The same order of progression occurs despite allowing phenotypic reversibility within our model. This is an important conceptual advance as it means mutations are not a necessary mechanism for phenotypic variation within tumour tissue; rather the model demonstrates that heritable, but potentially 
reversible, epigenetic changes may account for some of the phenotypic changes observed in cancer cells.

In order to examine the effects of parameter changes on system dynamics, we define a measure of the 'fitness' of a specific parameter set. Let 'invasive' be used to describe cells displaying all three heritable changes and, for a particular automaton, let $T$ denote the number of generations after which $95 \%$ of the cells in the system display the invasive phenotype. Thus $T$ is representative of the amount of time taken for full carcinogenesis to occur. Now let the development rate $R=T^{-1}$, where we take $R=$ 0 if $T \geqslant 5000$ (equivalent to approximately 20 years). Automata with a higher value of $R$ proceed more quickly through the carcinogenesis pathway.

In Fig. 5 we see how the development rate $R$ varies with changes in (a) glycolytic rate $k$, (b) acid resistance $h_{T}$ and (c) adaptation rate $p_{a}$. Using default parameters of $k=10$, $h_{T}=8.6 \times 10^{3}$ and $p_{a}=10^{-3}$, the three graphs show the effects of changing one of these parameters whilst keeping the other two fixed at their default value. Each data point is the mean value of $R$ calculated over fifty simulations, whilst the accompanying error bars show the standard errors of these means.

Fig. 5(a) shows a sharp transition from slow development to rapid development as the glycolytic rate $k$ is increased through a critical threshold value of $k \approx 3$. This transition occurs when the increase in ATP production and extracellular acidity due to upregulation of glycolysis is sufficient to give the invasive cell population a significant advantage over their untransformed counterparts. A similar bifurcation has been seen in other models looking at the role of acidity in tumour growth, whereby a transition from benign to malignant growth is seen when the cellular acid production rate increases through a critical point (Patel et al., 2001; Gatenby and Gawlinski, 1996, 2003; Smallbone et al., 2005). Increasing $k$ beyond 20 results in a slow monotonic decrease in the development rate. For such large values of $k$, acid accumulates to a degree unfavourable even to the resistant invasive cells, inducing auto-toxicity.

In Fig. 5(b) we see that initially the development rate increases sharply with increasing acid resistance, reaching a plateau at $h_{T} \approx 2 \times 10^{3}$. For large $h_{T}$, we find the benefits of increasing acid resistance are counteracted by clumps of acid-resistant non-glycolytic cells developing near the basement membrane, withstanding the progression of the invasive phenotype. In the microenvironment near the membrane, the non-glycolytic cells produce sufficient ATP and are extremely resistant to extracellular acidity; thus their invasive, glycolytic counterparts have only a small competitive advantage.

Finally, Fig. 5(c) shows that, as with the glycolytic rate $k$, there is a value of adaptation rate $p_{a}$ at which the development rate is optimal. Increasing the adaptation rate increases the diversity of the system. This leads to an increased chance of acquiring the invasive phenotype, whilst reducing the dominance of the main population in

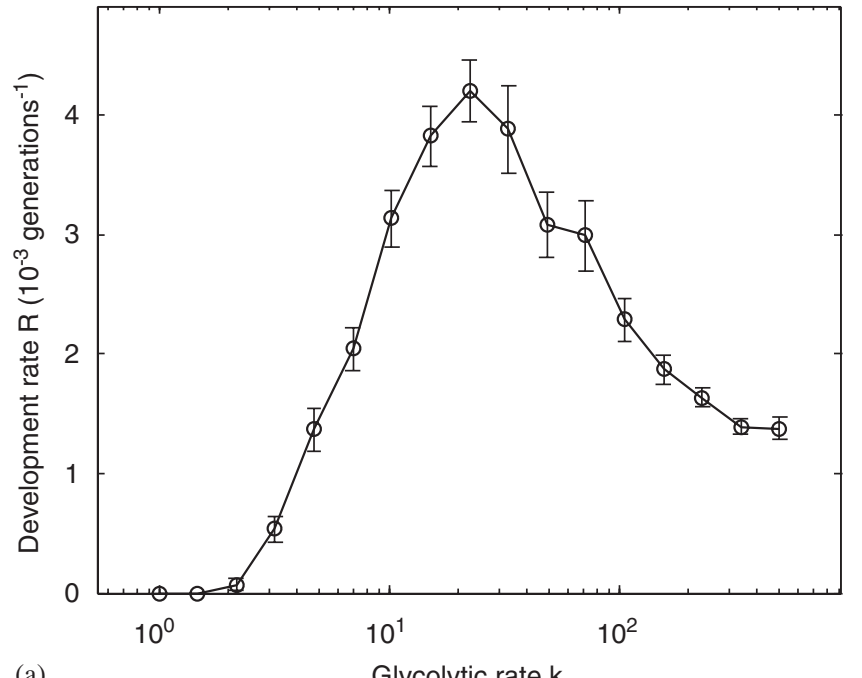

(a) Glycolytic rate $\mathrm{k}$

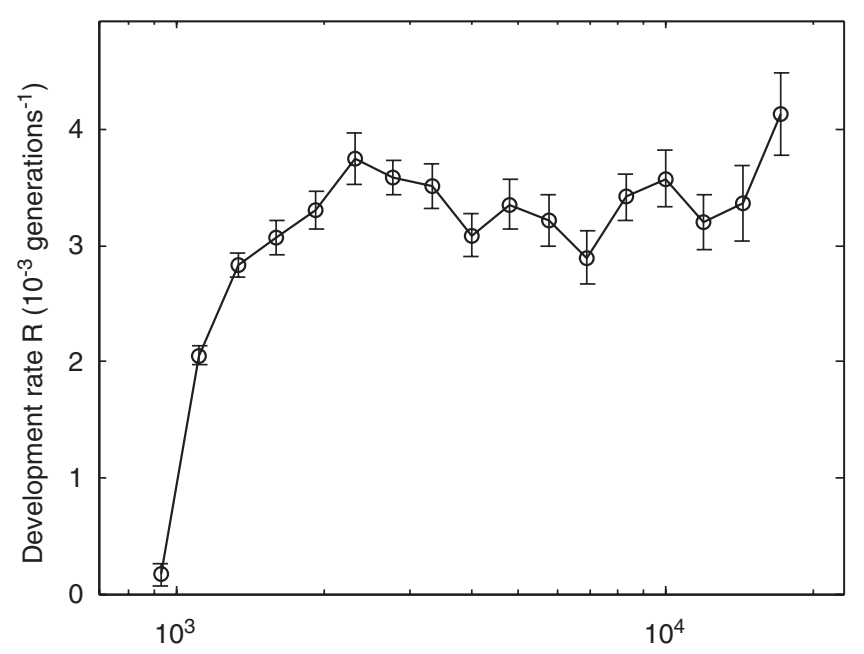

(b) Acid resistance $\mathrm{h}_{\mathrm{T}}$

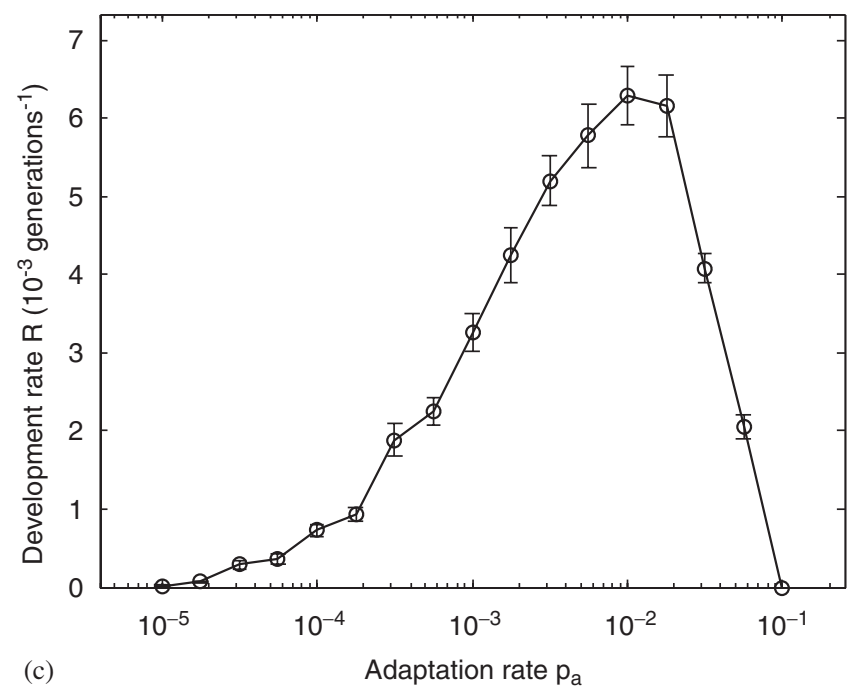

Fig. 5. Variation in the development rate $R$ with (a) glycolytic rate $k$, (b) acid resistance $h_{T}$ and (c) adaptation rate $p_{a}$. Default parameter values used are $k=10, h_{T}=8.6 \times 10^{3}$ and $p_{a}=10^{-3}$. Note that $k, h_{T}$ and $p_{a}$ are plotted on a log-scale. Each data point is the mean value of $R$ calculated over 50 simulations, whilst the accompanying error bars show the standard errors of these means. 
the system. This balance resolves itself with the maximum development rate occurring at $p_{a} \approx 10^{-2}$. The model's results here are consistent with the Eigen-Schuster quasispecies theory (Eigen, 1971; Schuster, 1994), namely that a critical mutation rate known as the 'error catastrophe' exists beyond which the genomic information is lost i.e. no Darwinian selection operates. Returning to Fig. 5(c), we see that this error catastrophe threshold falls at $p_{a} \approx 10^{-1}$.

\section{Discussion}

In this article, we address the evolutionary dynamics in carcinogenesis that promote aerobic glycolysis in the malignant phenotype and examine the potential role of abnormal glucose metabolism in formation of invasive cancers.

Carcinogenesis is a complex multi-step process governed by the interactions of heritable phenotypic variations with continuously changing environmental selection forces. The dynamics of carcinogenesis are often summarized as somatic evolution because they appear to be formally analogous to Darwinian selection in nature. Thus defined, the common appearance of a specific phenotype within different cancer populations must be the result of environmental selection and, therefore, must confer a significant growth advantage.

Since the pioneering work of Warburg (1930) nearly a century ago, experimental observations have consistently demonstrated that cancer cells, unlike their normal counterparts, utilize anaerobic pathways to metabolize glucose even in the presence of oxygen. The clinical importance of this phenotypic trait is suggested by FDGPET imaging, which demonstrates a several-fold increased glucose uptake in the vast majority of human primary and metastatic cancers. However, in the context of the evolutionary model of carcinogenesis, the competitive advantage of altered glucose metabolism is not immediately clear since it represents a highly inefficient means of energy production and results in significant acidosis of the tumour micro-environment.

The work presented here uses a hybrid cellular automaton approach to examine the role of the microenvironment in mediating the somatic evolution of cancer cells. Utilizing the fact that epithelial tumours evolve on mucosal surfaces separated from their blood supply by the intact basement membrane, we extend previous evolutionary modelling of carcinogenesis to explicitly include spatial parameters that accommodate these boundary conditions. This new modelling approach allows quantification of regional variations in the microenvironment in premalignant lesions.

We examine the hypothesis that upregulation of glycolysis represents an adaptation to hypoxia in premalignant lesions that develops as tumour cells grow into the lumen of the duct and away from their blood supply. This new phenotype, in turn, produces environmental acidosis which promotes additional adaptation to prevent acidinduced cell death. The phenotype that emerges from this sequence has a substantial, general proliferative advantage because it creates an environment that is toxic to its competitor but not to itself. This competitive advantage allows an invasive phenotype that permits penetration through the basement membrane and formation of a primary carcinoma.

Our results confirm the hypothesis that hypoxia and anoxia will be common in premalignant lesions such as DCIS or advanced colon polyps. In fact, we demonstrate that even early hyperplastic lesions will contain areas of hypoxia once tumour growth carries cells to more than a few cell layers beyond the basement membrane. Similarly, our results confirm that regional development of hypoxia will promote upregulation of anaerobic metabolism of glucose and subsequent development of extracellular acidosis. Finally, we find the acidic $\mathrm{pH}_{e}$ that develops from this sequence will, in some regions, result in cellular toxicity and, therefore, become a significant environmental selection factor that promotes resistant phenotypes.

Clearly, confirmation of the modelling results by direct measurement of regional variations in oxygen, glucose and $\mathrm{H}^{+}$concentrations in premalignant lesions will be difficult. However, our results are likely to be quite realistic since the work is based on well-established biological application of reaction diffusion models where the values of critical parameters are known. In fact, the potential for development of hypoxia within tissue was demonstrated mathematically by Krogh nearly 100 years ago (Krogh, 1919). The presence of hypoxia in tumour cells more than $100-150 \mu \mathrm{m}$ from a blood vessel has been demonstrated experimentally by many investigators since the pioneering work by Thomlinson and Gray (Dewhirst et al., 1994; Thomlinson and Gray, 1955). Finally, experimental measurement of perivascular oxygen and $\mathrm{pH}_{e}$ gradients that both qualitatively and quantitatively resemble our modelling results have been reported by Helmlinger et al. (1997).

Our results also demonstrate possible pathways in somatic evolution that may result as cellular populations acquire new, fitter phenotypes in response to local proliferative constraints caused by variations in microenvironmental properties. This allows explicit predictions regarding regional variations in phenotype in both premalignant lesions such as DCIS and early invasive cancers. This predicted phenotypic variability should be experimentally testable and we are encouraged that published studies have shown evidence of adaptation to hypoxia through increased expression of carbonic anhydrase IX in DCIS cells that are nearest the lumen (i.e. most distant from the basement membrane) (Wykoff et al., 2001).

Finally, our results suggest that tumour prevention strategies aimed at interrupting the hypoxia-glycolysis-acidosis cycle and the resulting cellular adaptations will delay or prevent transition from in situ to invasive cancer. 
For example, our results suggest that drugs that block the function of the $\mathrm{Na}^{+} / \mathrm{H}^{+}$antiport (such as amiloride) would likely inhibit the adoption of constitutive upregulation of aerobic glycolysis.

In summary, our study supports the hypothesis that regional variations in oxygen, glucose and $\mathrm{H}^{+}$levels drive the final stages of somatic evolution during carcinogenesis. We propose that the phenotypic adaptations to the sequence of hypoxia-glycolysis-acidosis are necessary to form an invasive cancer. For this reason, interruption will likely delay or prevent transition from in situ to invasive cancer.

\section{Acknowledgments}

K.S. and D.J.G. acknowledge the support of grant GR/ R96149/01 from the Engineering and Physical Sciences Research Council through the Oxford University Life Sciences Doctoral Training Centre. R.A.G. and P.K.M. acknowledge the support of NIH Grant U56CA113004 from the National Cancer Institute.

\section{Appendix A}

\section{A.1. Model of cellular metabolism}

Suppose the cell consumes glucose and oxygen at rates $\Phi_{G}$ and $\Phi_{C}$, respectively, and that all of the consumed glucose and oxygen is used to generate ATP under the two processes outlined above. Then, from Eq. (1), we are assuming $\Phi_{G} \geqslant \Phi_{C} / 6$. If this condition is satisfied, we may calculate the rates of ATP production $\Phi_{A}$ and lactic acid production $\Phi_{L}$ from Eqs. (1) and (2),

$\Phi_{A}=\frac{n_{A} \Phi_{C}}{6}+2\left(\Phi_{G}-\frac{\Phi_{C}}{6}\right)$

$\Phi_{L}=2\left(\Phi_{G}-\frac{\Phi_{C}}{6}\right)$.

The lactic acid produced by the cell partially disassociates into $\mathrm{H}^{+}$and lactate. These $\mathrm{H}^{+}$ions lower the $\mathrm{pH}$ of the extracellular space, inducing cellular toxicity. The rate of cellular $\mathrm{H}^{+}$production $\Phi_{H}$ is taken to be proportional to the rate of lactic acid production, $\Phi_{H}=k_{H} \Phi_{L}$, for some $k_{H}<1$. Note that the aerobic pathway also contributes to cellular acid production through hydration of $\mathrm{CO}_{2}$. However, this contribution is small - for each mole of ATP synthesized, anaerobic metabolism produces one mole of lactic acid, whilst aerobic metabolism produces only $\frac{1}{6}$ mole of $\mathrm{CO}_{2}$. As such we ignore this term, considering only the acid production in excess of the normal rate.

It remains to define the rates of cellular glucose and oxygen consumption $\Phi_{G}$ and $\Phi_{C}$. Whilst complex empirical functional forms for these rates are available (Casciari et al., 1992), here we assume that the rates follow simpler first-order dynamics,

$\Phi_{G}= \begin{cases}k_{N} G & \text { in a normal cell, } \\ k_{T} G & \text { in a glycolytic cell, }\end{cases}$

$\Phi_{C}=k_{C} C$,

where $G$ and $C$ denote the extracellular concentrations of glucose and oxygen, respectively, and $k_{T}>k_{N}$. Note that we assume that tumour cells do not significantly alter their rate of oxygen consumption during carcinogenesis, consistent with experimental observations (Ramanathan et al., 2005).

We non-dimensionalize Eqs. (5)-(8), to reduce the size of the parameter space. Let $G_{X}$ and $C_{X}$ denote the normal extracellular concentrations of glucose and oxygen, and suppose that under normal conditions, normal cells rely on aerobic respiration alone to produce energy. Then $k_{C} C_{X}=$ $6 k_{N} G_{X}$ and

$\phi_{g}= \begin{cases}g & \text { in a normal cell, } \\ k g & \text { in a glycolytic cell, }\end{cases}$

$\phi_{c}=c$

$\phi_{a}=c+n\left(\phi_{g}-c\right)$

$\phi_{h}=\phi_{g}-c$,

subject to the condition $\phi_{g} \geqslant c$, where

$g=\frac{G}{G_{X}}, \quad c=\frac{C}{C_{X}}, \quad \phi_{g}=\frac{\Phi_{G}}{k_{N} G_{X}}, \quad \phi_{c}=\frac{\Phi_{C}}{k_{C} C_{X}}$,

$\phi_{a}=\frac{\Phi_{A}}{n_{A} k_{N} G_{X}}, \quad \phi_{h}=\frac{\Phi_{H}}{2 k_{H} k_{N} G_{X}}, \quad n=\frac{2}{n_{A}}$,

$k=\frac{k_{T}}{k_{N}}$.

The non-dimensionalized model of cellular respiration relies on two parameters: $n=\frac{1}{18}$ and $k$. Given ranges $10^{-6} \mathrm{~s}^{-1}<k_{N}<5 \times 10^{-4} \mathrm{~s}^{-1}$ and $10^{-5} \mathrm{~s}^{-1}<k_{T}<10^{-3} \mathrm{~s}^{-1}$ (Kallinowski et al., 1988) for the rates of glucose consumption by normal and tumour cells, respectively, we assume $1<k<10^{3}$, i.e. that glycolytic cells may increase their glucose consumption by up to three orders of magnitude.

\section{A.2. Metabolite models}

Consider first the extracellular concentration of glucose, $G$. Note that the glucose diffusion time-scale ( $\sim$ minutes) is much shorter than the cellular proliferation time-scale ( $\sim$ days), and thus we may assume that $G$ is in diffusive equilibrium at all times. Then we have

$D_{G} \nabla^{2} G-\Phi_{G}=0$,

where $D_{G}$ is the (assumed constant) glucose diffusion coefficient. We non-dimensionalize Eq. (14), taking cell diameter as our length scale. Using (13),

$d_{g}^{2} \nabla_{\xi}^{2} g-\phi_{g}=0$ 
where $\xi=x / \Delta$ and $d_{g}=\sqrt{D_{G} / k_{N} \Delta^{2}}$. Given $D_{G}=5 \times$ $10^{-6} \mathrm{~cm}^{2} \mathrm{~s}^{-1}$ (Groebe et al., 1994) and taking $k_{N}=$ $5 \times 10^{-5} \mathrm{~s}^{-1}$, we find $d_{g}=1.3 \times 10^{2}$. In a spatially homogeneous system of normal cells, $d_{g} \log 2 \approx 90$ represents the number of cells away from the basement membrane at which the glucose concentration drops to half its normal level. In a system of glycolytic cells, where glucose is consumed at a higher rate, this distance falls to $d_{g} \log 2 / \sqrt{k}$.

Eq. (15) is solved using a finite-difference approximation

$g_{i+1, j}+g_{i-1, j}+g_{i, j+1}+g_{i, j-1}-\left(4+\delta_{i, j}\right) g_{i, j}=0$,

where $g_{i, j}$ refers to the glucose level of the $i-j$ th automaton element and $\delta_{i, j}$ depends on the element's occupancy

$\delta_{i, j}= \begin{cases}0 & \text { in a vacant cell, } \\ 1 / d_{g}^{2} & \text { in a normal cell, } \\ k / d_{g}^{2} & \text { in a glycolytic cell. }\end{cases}$

As boundary conditions, we assume that the glucose levels are fixed at their normal levels at the basement membrane (as the stroma is well-vascularized), zero flux at the edge furthest from the membrane (as there are no sources or sinks of glucose beyond this point), and periodic boundary conditions at the other two edges. Using the notation of Eq. (16), this may be written as

$g_{0, j}=1, \quad g_{M+1, j}=g_{M, j} \quad \forall j=1, \ldots, N$,

$g_{i, 0}=g_{i, N}, \quad g_{i, N+1}=g_{i, 1} \quad \forall i=1, \ldots, M$.

Eq. (16) holds $\forall i=1, \ldots, M$ and $\forall j=1, \ldots, N$ and is thus representative of a system of $M \times N$ linear algebraic equations in the unknown $g_{i, j}$. The equilibrium glucose field $g=\left(g_{i, j}\right)$ may then be found through simple matrix inversion.

The oxygen distribution around the tumour is found using the same method. In non-dimensional form we have

$d_{c}^{2} \nabla_{\xi}^{2} c-\phi_{c}=0$,

where $d_{c}=\sqrt{D_{C} / k_{C} \Delta^{2}}$ and $D_{C}$ is the oxygen diffusion coefficient. Given $k_{C}=9.41 \times 10^{-2} \mathrm{~s}^{-1}$ (Casciari et al., 1992 ) and $D_{C}=1.46 \times 10^{-5} \mathrm{~cm}^{2} \mathrm{~s}^{-1}$ (Nichols and Foster, 1994), we find $d_{c}=5 \ll d_{g}$. In stark contrast to glucose, oxygen supply is very limited due to its small relative diffusion rate, with areas of hypoxia developing within a few cells of the basement membrane. Note that, in order for the model to be well-defined, we require $\phi_{g} \geqslant c$ at each cell, for which it is sufficient that $g \geqslant c$ everywhere. This holds if $k \leqslant d_{g}^{2} / d_{c}^{2} \approx 700$ and as such we restrict our attention here to the parameter range $1<k \leqslant 500$.

The equilibrium oxygen field $c$ is found from Eq. (19) using the same technique as for glucose. Having determined the glucose and oxygen fields, we know their rates of consumption, $\phi_{g}$ and $\phi_{c}$, for each individual cell. Then, from Eq. (12), we may calculate the rate of cellular $\mathrm{H}^{+}$ production, $\phi_{h}$. Unlike glucose and oxygen, $\mathrm{H}^{+}$ions do not follow simple (Fickian) diffusion, as this would lead to charge separation. Rather, they diffuse in association with mobile buffering species such as bicarbonate, phosphate, or amino acids (Schornack and Gillies, 2003). However, their movement may be approximated by simple diffusion, with appropriate modification of the diffusion coefficient. Thus the $\mathrm{H}^{+}$distribution, $h$, is defined by

$\nabla_{\xi}^{2} h+\phi_{h}=0$,

where $h=\left(H-H_{X}\right) / H_{0}$ and $H_{0}=2 k_{H} k_{N} G_{X} \Delta^{2} / D_{H}$. Here $H$ is the extracellular concentration of $\mathrm{H}^{+}, H_{X} \equiv$ pH 7.25 its normal level and $D_{H}$ its effective diffusion coefficient. This specific form for the scaling factor $H_{0}$ is chosen to remove the diffusion coefficient from Eq. (20). Given parameter values $D_{H}=1.08 \times 10^{-5} \mathrm{~cm}^{2} \mathrm{~s}^{-1}$ and a maximum tumour acid production rate of $10^{-4} \mathrm{mM} \mathrm{s}^{-1}$ (Patel et al., 2001), and assuming this is equivalent to our maximum non-dimensionalized rate of $\phi_{h}=500$, we may estimate $H_{0}=1.1 \times 10^{-7} \mathrm{mM}$.

Eq. (20) is solved as before using a finite-difference approximation, with the difference in this case that $h=0$ is the normal level at the basement membrane.

\section{References}

Alarcón, T., Byrne, H.M., Maini, P.K., 2003. A cellular automaton model for tumour growth in an inhomogeneous environment. J. Theor. Biol. $225,257-274$.

Anderson, A.R.A., 2005. A hybrid mathematical model of solid tumour invasion: the importance of cell adhesion. Math. Med. Biol. 22, 163-186.

Bernards, R., Weinberg, R.A., 2002. A progression puzzle. Nature 418 , 823.

Casciari, J.J., Sotirchos, S.V., Sutherland, R.M., 1992. Variations in tumor cell growth rates and metabolism with oxygen concentration, glucose concentration, and extracellular pH. J. Cell. Physiol. 151, 386-394.

Czernin, J., Phelps, M.E., 2002. Positron emission tomography scanning: current and future applications. Annu. Rev. Med. 53, 89-112.

Dewhirst, M.W., Secomb, T.W., Ong, E.T., Hsu, R., Gross, J.F., 1994. Determination of local oxygen consumption rates in tumors. Cancer Res. 54, 3333-3336.

Di Chiro, G., Hatazawa, J., Katz, D.A., Rizzoli, H.V., De Michele, D.J., 1987. Glucose utilization by intracranial meningiomas as an index of tumor aggressivity and probability of recurrence: a PET study. Radiology 164, 521-526.

Eigen, M., 1971. Self-organization of matter and the evolution of biological macro-molecules. Naturwissenschaften 58, 465-523.

Fearon, E.R., Vogelstein, B., 1990. A genetic model for colorectal tumorigenesis. Cell 61, 759-767.

Gambhir, S.S., 2002. Molecular imaging of cancer with positron emission tomography. Nat. Rev. Cancer 2, 683-693.

Gatenby, R.A., Gawlinski, E.T., 1996. A reaction-diffusion model of cancer invasion. Cancer Res. 56, 5745-5753.

Gatenby, R.A., Gawlinski, E.T., 2003. The glycolytic phenotype in carcinogenesis and tumor invasion: insights through mathematical models. Cancer Res. 63, 3847-3854.

Gatenby, R.A., Gillies, R.J., 2004. Why do cancers have high aerobic glycolysis? Nat. Rev. Cancer 4, 891-899.

Gatenby, R.A., Maini, P.K., 2003. Mathematical oncology: cancer summed up. Nature 421, 321.

Gatenby, R.A., Gawlinski, E.T., Tangen, C., Flanigan, R.C., 2002. The possible role of post operative azotemia in enhanced survival of 
patients with metastatic renal cancer following cytoreductive nephrectomy. Cancer Res. 62, 5218-5222.

Groebe, K., Erz, S., Mueller-Kleiser, W., 1994. Glucose diffusion coefficients determined from concentration profiles in EMT6 tumor spheroids incubated in radioactively labeled L-glucose. Adv. Exp. Med. Biol. 361, 619-625.

Helmlinger, G., Yuan, F., Delian, M., Jain, R.K., 1997. Interstitial pH and $\mathrm{pO}_{2}$ gradients in solid tumors in vivo: high-resolution measurements reveal a lack of correlation. Nat. Med. 3, 177-182.

Kallinowski, F., Vaupel, P., Runkel, S., Berg, G., Fortmeyer, H.P., Baessler, K.H., Wagner, K., 1988. Glucose uptake, lactate release, ketone body turnover, metabolic milieu and $\mathrm{pH}$ distributions in human cancer xenograpts in nude rats. Cancer Res. 48, 7264-7272.

Komarova, N.L., 2005. Mathematical modeling of tumorigenesis: mission possible. Curr. Opin. Oncol. 17, 39-43.

Krogh, A., 1919. The number and distribution of capillaries in muscles with calculations of the oxygen pressure head necessary for supplying the tissue. J. Physiol. 52, 409-415.

Kunkel, M., Reichert, T.E., Benz, P., Lehr, H., Jeong, J., Wieand, S., Bartenstein, P., Wagner, W., Whiteside, T., 2003. Overexpression of Glut-1 and increased glucose metabolism in tumors are associated with a poor prognosis in patients with oral squamous cell carcinoma. Cancer 97, 1015-1024.

Melicow, M.M., 1982. The three-steps to cancer: a new concept of carcinogenesis. J. Theor. Biol. 94, 471-511.

Nichols, M.G., Foster, T.H., 1994. Oxygen diffusion and reaction kinetics in the photodynamic therapy of multicell tumour spheroids. Phys. Med. Biol. 39, 2161-2181.
Patel, A.A., Gawlinski, E.T., Lemieux, S.K., Gatenby, R.A., 2001. A cellular automaton model of early tumor growth and invasion. J. Theor. Biol. 213, 315-331.

Racker, E., 1974. History of the Pasteur effect and its pathobiology. Mol. Cell. Biochem. 5, 17-23.

Ramanathan, A., Wang, C., Schreiber, S.L., 2005. Perturbational profiling of a cell-line model of tumorigenesis by using metabolic measurements. Proc. Natl Acad. Sci. USA 102, 5992-5997.

Rohzin, J., Sameni, M., Ziegler, G., Sloane, B.F., 1994. Pericellular pH affects distribution and secretion of cathepsin B in malignant cells. Cancer Res. 54, 6517-6525.

Schornack, P.A., Gillies, R.J., 2003. Contributions of cell metabolism and $\mathrm{H}^{+}$diffusion to the acidic $\mathrm{pH}$ of tumors. Neoplasia 5, 135-145.

Schuster, P., 1994. How do RNA molecules and viruses explore their worlds? Complexity: Metaphors, Models and Reality. AddisonWesley, Reading, MA, pp. 383-418.

Smallbone, K., Gavaghan, D.J., Gatenby, R.A., Maini, P.K., 2005. The role of acidity in solid tumour growth and invasion. J. Theor. Biol. $235,476-484$.

Thomlinson, R.H., Gray, L.H., 1955. The histological structure of some human lung cancers and the possible implications for radiotherapy. Br. J. Cancer 9, 539-549.

Warburg, O., 1930. The Metabolism of Tumours. Constable Press, London.

Wykoff, C.C., Beasley, N., Watson, P.H., Campo, L., Chia, S.K., English, R., Pastorek, J., Sly, W.S., Ratcliffe, P., Harris, A.L., 2001. Expression of the hypoxia-inducible and tumor-associated carbonic anhydrases in ductal carcinoma in situ of the breast. Am. J. Pathol. 158, 1011-1019. 